\title{
miRNA-320a inhibits tumor proliferation and invasion by targeting c-Myc in human hepatocellular carcinoma
}

This article was published in the following Dove Press journal:

OncoTargets and Therapy

15 February 2017

Number of times this article has been viewed

\author{
Fei Xie ${ }^{1, *}$ \\ Yuncang Yuan ${ }^{1, *}$ \\ Luyang $\mathrm{Xie}^{2, *}$ \\ Pengzhan Ran' \\ Xudong Xiang ${ }^{3}$ \\ Qionglin Huang' \\ Guoxiang $\mathrm{Qi}^{\prime}$ \\ Xiaopeng Guo' \\ Chunjie Xiao' \\ Shangyong Zheng' \\ 'School of Medicine, Yunnan \\ University, Kunming, Yunnan, \\ ${ }^{2}$ Department of Stomatology, Shanghai \\ Tenth People's Hospital, Shanghai, \\ ${ }^{3}$ Department of Gastrointestinal \\ Surgery, The Third Affiliated Hospital \\ of Kunming Medical University, \\ Kunming, Yunnan, People's Republic \\ of China \\ *These authors contributed equally \\ to this work
}

Background: Downregulated expression levels of microRNA-320a (miR-320a) were found in primary breast cancers and colorectal cancer. Previous findings indicated that miRNA-320a may involve in the cancer development. In this study, we explored the roles of miR-320a by targeting c-Myc in the tumor growth of hepatocellular carcinoma (HCC).

Methods: Quantitative reverse-transcription polymerase chain reaction (qRT-PCR) was performed to detect the expression of miR-320a in $50 \mathrm{HCC}$ tissues and four HCC cells. Luciferase reporter assay was conducted to confirm the direct downstream target of miR-320a in HEK-293 cells. The effect of miR-320a on endogenous c-Myc expression was investigated by transfecting miR-320a mimics into HepG2 and QGY-7703 cell lines. The c-Myc and miR-320a expressions were analyzed by immunohistochemistry (IHC) and qRT-PCR in the same HCC tissues. Furthermore, the biological functional correlation of miR-320a with c-Myc was determined by studying the effect of miR-320a mimics or c-Myc small interfering RNA (siRNA) on HCC cell proliferation and invasion.

Results: The expression of miR-320a was downregulated in $50 \mathrm{HCC}$ tissues and $4 \mathrm{HCC}$ cells. Luciferase assay revealed that c-Myc is a direct target of miR-320a. IHC and Western blot analysis showed that the c-Myc expression was inhibited by miR-320a in HCC tissues and cell lines. Upregulation of miR-320a suppressed the HCC cell proliferation and invasion capacity induced by inhibiting c-Myc, and the results were consistent with the effects of c-Myc siRNA on tumor suppression. These results revealed that miRNA-320a inhibits tumor proliferation and invasion by targeting c-Myc in HCC cells.

Conclusion: Our results showed that miR-320a functions as a tumor suppressor in HCC. By targeting c-Myc directly, miR-320a inhibits the HCC cell growth. Our studies provide evidence of miR-320a as a potentially target for HCC treatment.

Keywords: miR-320a, hepatocellular carcinoma, c-Myc, tumor suppressors

\section{Introduction}

MicroRNAs (miRNAs) are small noncoding RNA molecules that are often located in genomic breakpoint regions and play important roles in regulating diverse biological processes. Increasing evidence indicates that some miRNAs function as tumor suppressors or oncogenes in the tumor development. ${ }^{1-3}$ The gene expressions are found to be regulated by miRNAs by targeting the $3^{\prime}$ untranslated regions of mRNA specifically, and many targeting genes may be controlled by one specific miRNA. Translational level or mRNA stability is influenced by binding of miRNAs to target mRNAs. The cancer invasion effect of miRNAs has been identified in several cancer types, for example gastric cancer, ${ }^{4}$ hepatocellular carcinoma $(\mathrm{HCC}),{ }^{5}$ colorectal cancer $(\mathrm{CRC}),{ }^{6}$ pancreatic
Correspondence: Chunjie Xiao; Shangyong Zheng

School of Medicine, Yunnan University,

2 Cuihu North Road, Kunming,

Yunnan 65009I, People's Republic

of China

Tel +8618208890689

Fax +86 87I 65034358

Email chjxiao@ynu.edu.cn;

shangyong@ynu.edu.cn
OncoTargets and Therapy 2017:10 885-894 Dovepress http::/dx.doi.org/10.2147/0TTS122992 (c) (1) (5) 2017 Xie et al. This work is published and licensed by Dove Medical Press Limited. The full terms of this license are available at https://www.dovepress.com/terms.php cc) ${ }_{\mathrm{BY}} \mathrm{NC}$ and incorporate the Creative Commons Attribution - Non Commercial (unported, v3.0) License (http://creativecommons.org/licenses/by-n/3.0/). By accessing the work you hereby accept the Terms. Non-commercial uses of the work are permitted without any further permission from Dove Medical Press Limited, provided the work is properly attributed. For permission for commercial use of this work, please see paragraphs 4.2 and 5 of our Terms (https://www.dovepress.com/terms.php). 
cancer, and so on. ${ }^{7}$ Most studies have analyzed the association between miRNAs and invasion in vitro and mouse models. Differential miRNAs are only able to be identified in primary tumor tissues versus normal tissue in tissue level.

HCC, which accounts for $\sim 700,000$ deaths per year, is the third leading cause of cancer-related death in the world. ${ }^{8,9}$ Great efforts on the HCC biological mechanism studies had been taken to improve treatment therapy. A number of studies observed abnormal miRNA expressions in HCC. Previous studies showed that HCC cell growth were regulated by miRNAs, such as miR-101, ${ }^{10} \mathrm{miR}-221,{ }^{11} \mathrm{miR}-224,{ }^{12}$ miR-195, ${ }^{13} \mathrm{miR}-122,{ }^{14} \mathrm{miR}-223,{ }^{15} \mathrm{miR}-21,{ }^{16}$ and miR-199, ${ }^{17}$ and apoptosis, migration, and invasion of HCC cell were regulated by them as well. miRNAs involve in the HCC progression, diagnosis, and management. ${ }^{9}$ There are studies that suggest that HCC tumorigenesis may be caused by miRNA abnormal expression. Thus, extensive investigations are needed to identify the function of these miRNAs on HCC development, and some potential miRNAs may be used as new biomarkers for the diagnosis and prognosis of HCC. ${ }^{9,18-20}$

MiR320a downregulation was observed in colon cancer, ${ }^{21}$ breast cancer $(\mathrm{BC}),{ }^{22,23}$ bladder carcinoma, ${ }^{24}$ and gastric cancer, ${ }^{25}$ Therefore, we hypothesized that miR-320a may involve in the tumorigenesis and tumor development, and here we focused on the mechanism pathway studies of miR-320a in HCC tumorigenesis and development.

In this study, the expression of miRNA-320a was examined in HCC tissues and HCC cells, such as BEL-7402, SMMC-7721, HSPG2, and QGY-7703 cell lines compared with normal liver tissues and L02 cell line. Then, we analyzed the miRNA expression profiles to provide a hypothesis of the possible target toward miRNA-320a. Next, we investigated the correlation of miRNA-320a with the potential target gene. Finally, further investigations were conducted to study the functional mechanism of the miRNA-320a in HCC cells. Our novel findings suggested that miR-320a may be a potential target or biomarker for HCC diagnosis and therapy.

\section{Methods}

\section{Ethical statements}

Our study was approved by the ethical committee of the Yunnan University School of Medicine. Written informed consent was obtained from all participants for this study.

\section{Samples}

Fifty paired HCC and adjacent nontumor specimens used in this study were obtained from the Department of Thoracic
Surgery, Third Affiliated Hospital of Kunming Medical University (Kunming, China). Five normal liver tissues were collected from adjacent liver tissues of contusion and laceration in traumatic liver injury patients. All tissue samples were flash-frozen in liquid nitrogen immediately after collection and stored at $-80^{\circ} \mathrm{C}$ until use. Both tumor and nontumor samples were confirmed by pathological examination. No patients received chemotherapy or radiotherapy prior to surgery.

\section{Cell culture}

In this study, HCC cell lines (BEL-7402, HepG2, QGY-7703, SMMC-7721, L02) were purchased from the cell bank of the Chinese Academy of Sciences, and they were cultured in Dulbecco's Modified Eagle's Medium (DMEM; Sigma-Aldrich, St Louis, MO, USA) supplemented with $10 \%$ (vol/vol) fetal bovine serum (FBS). HEK293 cells were purchased from American Type Culture Collection (Manassas, VA, USA), and cultured in DMEM containing 10\% (vol/vol) FBS. All cells were maintained at $37^{\circ} \mathrm{C}$ with $5 \% \mathrm{CO}_{2}$.

\section{RNA isolation and qRT-PCR}

Total RNA was isolated from HCC tissues, adjacent nontumor tissues, and BEL-7402, HepG2, QGY-7703, SMMC7721, and HEK293 cell lines using Trizol according to the manufacturer's instructions. QRT-PCR assay was performed as described previously. ${ }^{26} \mathrm{U} 6$ small RNA was used as an internal control for normalization. All primers used in this study were listed in Table 1.

\section{Western blot analysis}

To determine protein expression levels, proteins form cell lysates were separated by $10 \%$ sodium dodecyl sulfatepolyacrylamide gel and transferred to nitrocellulose membrane (Bio-Rad, Hercules, CA, USA). The membrane was blocked with $5 \%$ nonfat milk, then incubated with anti- $\beta$ Actin antibody (Sigma, CA, USA) or anti-c-Myc antibody (Santa Cruz, CA, USA), followed by goat anti-mouse secondary antibody (Pierce, IL, USA). Finally, the protein bands were detected with enhanced chemiluminescence reagents following the Vendor protocol (Pierce).

\section{Oligonucleotides transfection}

RNA oligos used in this study were chemically synthesized by Genepharma (Shanghai, China). The human miR-320a mimics sequence was $5^{\prime}$-AAA AGC UGG GUU GAG AGG GCG A-3'. The sequence for negative control was $5^{\prime}$-CAG UAC UUU UGU GUA GUA CAA- $3^{\prime}$. The oligonucleotides for c-Myc siRNA were 5'-AAC GUU AGC UUC ACC AAC 
Table I Primer sequences used for qRT-PCR

\begin{tabular}{ll}
\hline Name & Primer sequence \\
\hline U6 F & 5'-GTGCTCGCTTCGGCAGCACATATAC-3' \\
U6 R & 5'-AAAAATATGGAACGCTTCACGAATTTG-3' \\
miR-320a F & 5'-AAAAGCTGGGTTGAGAGGGCG-3' \\
c-Myc (A) F & 5'-AAAACTAGTCGACGATGCCCCTCAACG \\
& TTAGC-3' \\
c-Myc (A) R & 5'-AAAAAGCTTGGTCGGCCGTGGAGAAG \\
& CTCCC-3' \\
c-Myc (B) F & 5'-AAAACTAGTAGCTGGAGATGGTGACCG \\
& AGCTGC-3' \\
c-Myc (B) R & 5'-AAAAGCTTAGTCTTGCGAGGCGCAGGA \\
& CTTGGG-3' \\
c-Myc (A+B) F & 5'-AAAACTAGTCGACGATGCCCCTCAACG \\
& TTAGC-3' \\
c-Myc (A+B) R & 5'-AAAAGCTTAGTCTTGCGAGGCGCAGGA \\
& CTTGGG-3' \\
\hline
\end{tabular}

Abbreviations: $F$, forward primer; miR-320a, microRNA-320a; $R$, reverse primer.

ATT-3' (sense) and 5'-UGU UGG UGA AGC UAA CGU UTT-3' (antisense), and the sequence of control siRNA was 5'-UUC UCC GAA CGU GUC ACG UTT-3' (sense) and 5'-ACG UGA CAC GUU CGU AGA ATT-3' (antisense). The oligonucleotide transfections were conducted by using INTERFERin reagent according to the manufacturer's protocol (Polyplus-transfection).

\section{Luciferase assay}

Luciferase reporter plasmid was created through cloning human c-Myc sequence into pMIR-Report construct (Ambion, Austin, TX, USA). C-Myc sequence contains the potential miR-320a binding site. C-Myc fragment A (from 566 to 850), c-Myc fragment B (from 851 to 1,207), and c-Myc fragment A+B (from 566 to 1,207) were amplified using PCR. (The primers are listed in Table 1.) The luciferase reporter assay was performed as previously, ${ }^{26}$ and cotransfection of $50 \mathrm{nM}$ miRNA mimics or negative control, $100 \mathrm{ng}$ firefly luciferase reporter, and $20 \mathrm{ng}$ pRL-TK vector (Promega, Madison, WI, USA) into the HEK293 cells was performed by the JetPRIME reagent (Invitrogen) following manufactory's protocol. Twenty-eight hours after transfection, cells were harvested and analyzed using DualLuciferase Reporter Assay System (Promega).

\section{Cell proliferation assay}

Cells were plated in 96-well plates (Corning Co, Corning, NY, USA) with 4,000 cells in $100 \mu \mathrm{L}$ per well and incubated for $72 \mathrm{~h}$ after transfection with miR-30a mimics or mimics $\mathrm{NC}$, the and cell viability was evaluated by the MTT assay (Promega) according to the manufacturer's instructions. MTT reagent solution was dispensed into each well and incubated at $37^{\circ} \mathrm{C}$ in $5 \% \mathrm{CO}_{2}$ for $4 \mathrm{~h}$. The absorbance was then read at $490 \mathrm{~nm}$ in a microplate absorbance reader. Cell proliferation was assessed every $24 \mathrm{~h}$. Triplicate wells were measured for cell viability in each treatment group.

\section{Invasion assays}

The invasive ability of HCC cells was determined using 24-well transwell chambers coated with Matrigel (BD Pharmingen, San Jose, CA, USA). Transfected cells in serumfree medium were seeded at $5 \times 10^{4}$ in the upper compartment of chamber. After the chamber was incubated at $37^{\circ} \mathrm{C}$ for $24 \mathrm{~h}$, cells that migrated to the underside of the membrane were fixed with 4\% paraformaldehyde (Sigma-Aldrich), stained with crystal violet (Beyotime, Shanghai, China), imaged and counted with a microscope (Leica, UK). All experiments were performed three times.

\section{Immunohistochemistry}

In this study, IHC staining of the HCC samples was performed according to the protocol described previously. ${ }^{26}$ Xylene was used to dewax the paraffin-embedded tissue sections, and the deparaffinized sections were rehydrated using ethanol. Then, epitope retrieval was induced by in citrate buffer ( $\mathrm{pH}$ 6.0). Anti-c-Myc antibody was used to identify C-Myc expression (Santa Cruz, CA, USA).

\section{Statistical analysis}

Continuous variables were expressed as mean \pm SEM. The two-tailed $t$-test was used between groups. In all cases, $P<0.05$ was considered as statistically significant.

\section{Results MiR-320a was downregulated in HCC samples and cell lines}

To determine the expression of miR-320a in HCC samples and cell lines, total RNA was extracted from HCC tissues, adjacent nontumor tissues, normal liver cell line, and HCC cell lines; quantitative reverse-transcription polymerase chain reaction (qRT-PCR) was used to analyze the expression levels of miR-320a, and normalized against an endogenous control (U6 RNA). MiR-320a was decreased significantly in HCC tissues versus adjacent nontumor tissues (Figure 1A). At the meantime, miR-320a was downregulated in four HCC cell lines compared with normal liver tissues and normal liver cell line (Figure 1B). Thus, these data demonstrated that the expression of miR-320a was downregulated in HCC samples and $\mathrm{HCC}$ cell lines. 

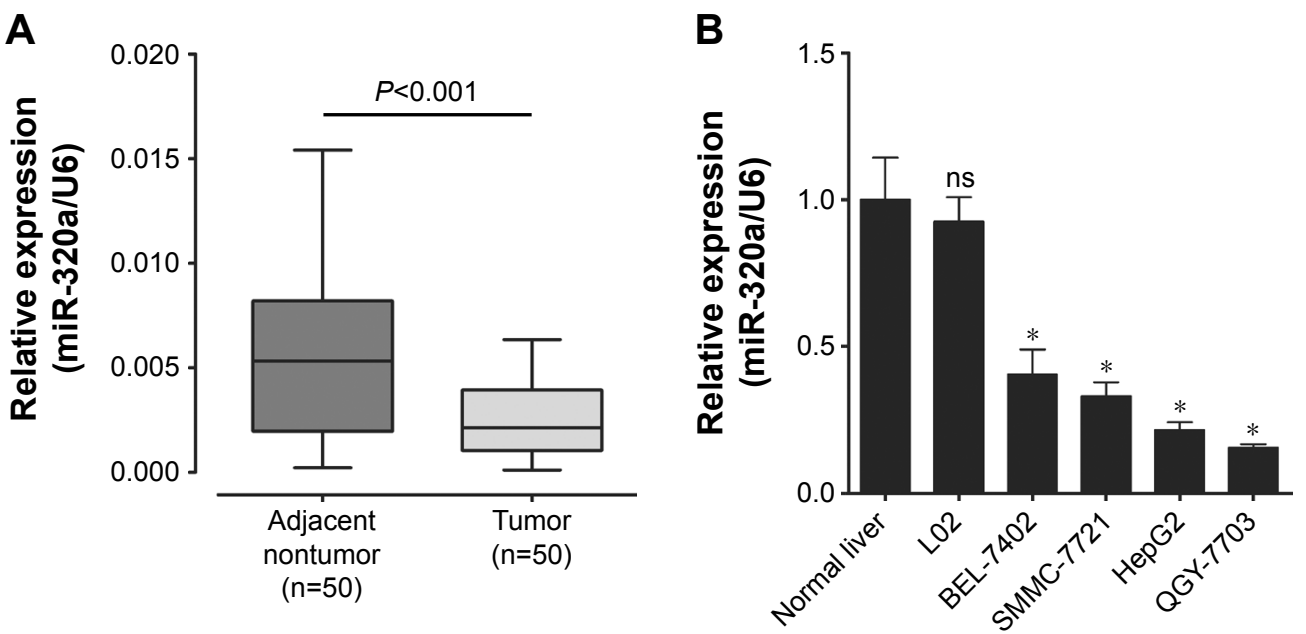

Figure I miR-320a relative expression in HCC samples and cell lines.

Notes: (A) miR-320a levels were accessed by qRT-PCR in HCCs and their adjacent nontumor tissues. Small nuclear RNA U6 was used as an internal control. (B) The expression of miR-320a was measured in five normal liver tissue, one normal cell line and four HCC cell lines by qRT-PCR, and the expression levels of miR-320a were normalized to U6. Data are presented as the mean \pm standard deviation $(n=3)$. Results were obtained in three replicates. $* P<0.05$.

Abbreviations: HCC, hepatocellular carcinoma; miR-320a, microRNA-320a; qRT-PCR, quantitative reverse-transcription polymerase chain reaction; ns, nonsignificant.

\section{C-Myc mRNA contains two target sequences for miR-320a}

Previous studies showed that miRNAs function by inhibiting target genes. To find the target gene of miR-320a correlated with HCC pathogenesis, the newly published CLASH data were further analyzed. ${ }^{27}$ Almost 514 genes were found to be targeted by miR-320a in HEK293 cells. We focused on gene c-Myc among these 514 genes, and there are two potential targeting sequences for miR-320a within the coding DNA sequence of c-Myc mRNA (Figure 2A). These data showed that c-Myc may be a potential target of miR-320a in HCC cells.

A

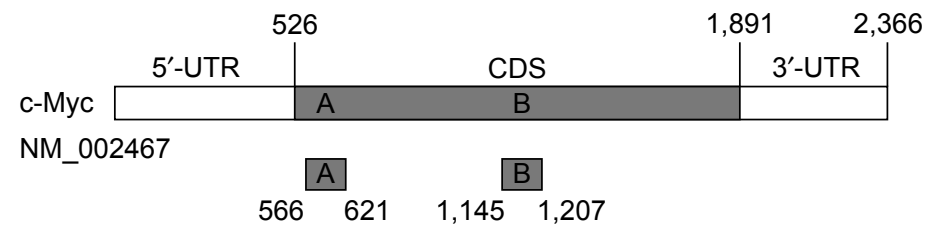

B

\begin{tabular}{|c|c|c|}
\hline Luciferase & & Empty \\
\hline Luciferase & A & c-Myc A \\
\hline Luciferase & B & C-Myc B \\
\hline Luciferase & $A$ & C-Myc A \\
\hline
\end{tabular}

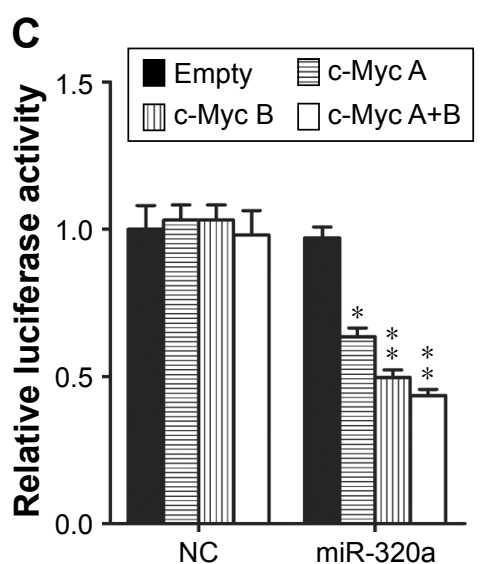

Figure 2 miR-320a directly binds to the coding DNA sequence of c-Myc.

Notes: (A) Schematic representation of the c-Myc messenger RNA with two putative miR-320a-binding sites in the CDS. (B) Schematic representation of luciferase reporter constructs. Two sequences including A or B sites were cloned into the luciferase reporter plasmid individually or in combination. (C) Luciferase reporter assay to verify the activity of miR-320a upon the target site of c-Myc. HEK-293 cells were transfected with luciferase reporter plasmid, miR-320a mimics, or NC. The firefly luciferase activity was normalized to the Renilla luciferase activity, and the normalized luciferase activity of the negative control was set as relative luciferase activity 1 . Data are shown as mean \pm standard deviation $(n=3)$. Results were obtained in three replicates. ${ }^{*} P<0.05$, $* * P<0.0$ I.

Abbreviations: CDS, coding DNA sequence; DNA, deoxyribonucleic acid; NC, negative control; miR-320a, microRNA-320a; UTR, untranslated regions. 


\section{C-Myc is a potential target of miR-320a in $\mathrm{HCC}$ cells}

There are data that showed that c-Myc may be a potential target of miR-320a; however, the correlation of c-Myc with miR-320a has not been clarified yet in HCC cells. To identify whether the c-Myc gene expression is regulated by miR-320a, luciferase reporter assay was performed in HEK-293 cells. First, we created luciferase reporter plasmid. Two sequences including two potential targeting sequences were individually or in combination cloned into luciferase reporter plasmid (Figure 2B), and miR-320a mimics or negative control was cotransfected into HEK293 cells for $48 \mathrm{~h}$. Next, the luciferase activity was detected using Dual-Luciferase Reporter Assay. The results demonstrated that the reporter plasmid with targeting sequence of $\mathrm{c}-\mathrm{Myc}$ mRNA lead to decrease of luciferase activity significantly in cells transfected with miR-320a compared with nonsequence of c-Myc reporter plasmid (Figure 2C). Hence, our findings proved that c-Myc is a direct target of miR-320a in HCC cells.

\section{MiR-320a inhibited c-Myc expression in HCC cells}

To determine the effect of miR-320a on c-Myc expression in HCC cell lines, HCC cell lines were transiently transfected with miR-320a mimics or negative control, and HCC cell lines showed low miR-320a expression. QRT-PCR was used to examine the expression of miR-320a. The results showed that miR-320a mimics transfection increased miR320a expression in HepG2 and QGY-7703 cells compared with the negative control (Figure 3A). Then, the c-Myc protein level was verified by Western blotting. Our results demonstrated that the expression of c-Myc was consistently and substantially downregulated by miR-320a upregulation (Figure 3B). Consistently, Immunohistochemistry (IHC) assay demonstrated low miR-320a expression but high c-Myc expression in HCC tissues, whereas the normal liver tissues with high miR-320a expression showed lower c-Myc expression (Figure 3C). Taken together, these findings proved that the c-Myc expression is upregulated by low expression of miR-320a in HCC cells.

\section{MiR-320a inhibited HCC cells proliferation and invasion by repressing c-Myc expression}

To examine the effects of miR-320a on HCC cells' function through targeting c-Myc, we designed the small interfering
RNA (siRNA) for c-Myc (c-Myc siRNA), which induced decrease of c-Myc expression at the protein levels in HepG2 and QGY-7703 cells, as shown in Figure 4A and B. At the same time, HepG2 and QGY-7703 cells were transfected with miR-320a mimics; we observed a similar decrease of c-Myc expression, as shown in Figure 4A and B. Next, the effect of miR-320a by targeting c-Myc on HCC cell viability was detected.

To identify the effects of miR-320a on HCC cell viability, 3-(4,5-dimethylthiazol-2-yl)-2,5-diphenyl tetrazolium bromide (MTT) assays were conducted, and the results showed that the proliferation ability of HepG2 (Figure 4C) and QGY-7703 cells (Figure 4D) transfected with miR-320a mimics were decreased obviously after $72 \mathrm{~h}$ compared with their respective controls. Similarly, we found that c-Myc siRNA reduced the proliferation ability of HepG2 (Figure 4C) and QGY-7703 cells (Figure 4D), and the effect of c-Myc siRNA on cell proliferation was consistent with that of miR-320a in HepG2 and QGY-7703 cells.

Next, the effect of miR-320a by targeting c-Myc on invasion capacity in $\mathrm{HCC}$ cells was detected. Invasion assay showed that invasion capacity of HepG2 (Figure 5A) and QGY-7703 cells (Figure 5B) transfected with miR-320a mimics was significantly decreased compared with their respective controls. Similarly, we found that c-Myc siRNA reduced the invasion capacity in HepG2 (Figure 5A) and QGY-7703 cells (Figure 5B), and the effect of c-Myc siRNA on cell invasion was consistent with that of miR-320a mimics in HepG2 and QGY-7703 cells. These findings suggested that miR-320a inhibits the invasion of HCC cells through targeting c-Myc.

\section{Discussion}

miRNAs are proved to play key roles in gene regulation by multiple studies. Previous study found that the expression of miR-320a is upregulated significantly in the peripheral blood of coronary heart disease. ${ }^{28}$ However, the expression of miRNA-320a was found to be downregulated in CRC, ${ }^{29}$ primary $\mathrm{BC},{ }^{30}$ and intrahepatic cholangiocarcinoma. ${ }^{31}$ In our present study, we found that miR-320a was significantly repressed in HCC tissues and HCC cell lines.

Previous studies showed that miRNAs could conduct their tumor repression function by inhibiting correlated target genes. In this study, to find the target gene of miR-320a correlated with HCC pathogenesis, miRNA expression profiles analysis was used and showed that 514 genes were targeted by miR-320a in HEK293 cells. We focused on c-Myc gene, which plays an important role in the mitogen-activated 
A

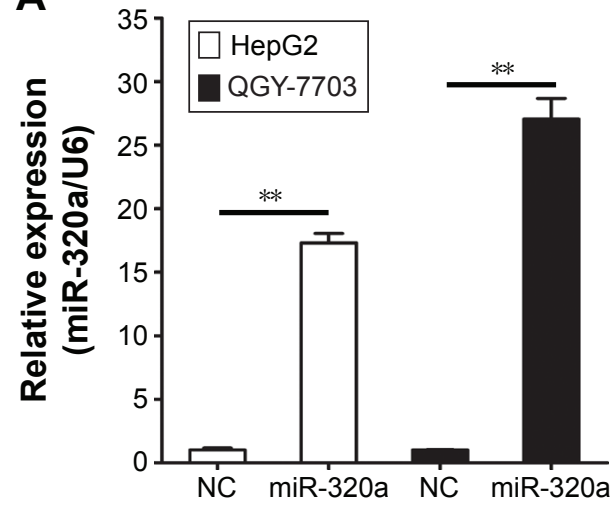

B

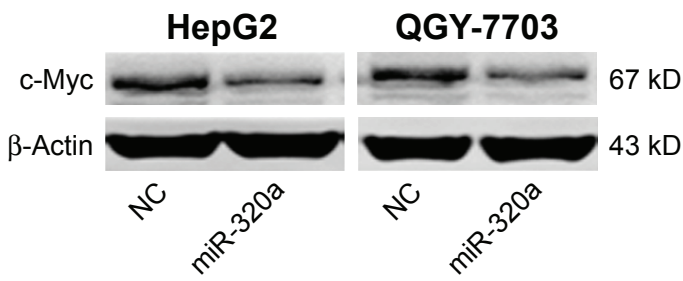

C

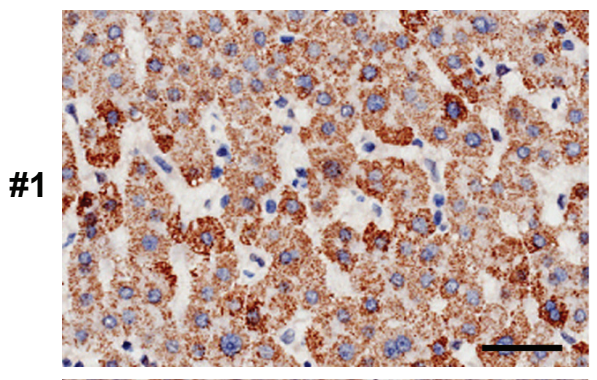
Tumor

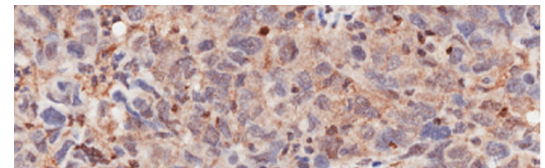

\#2

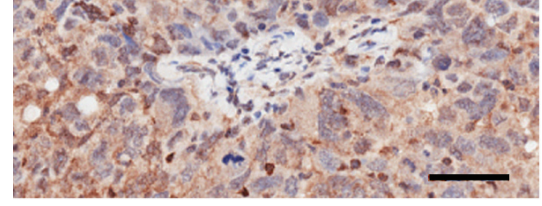

\#3

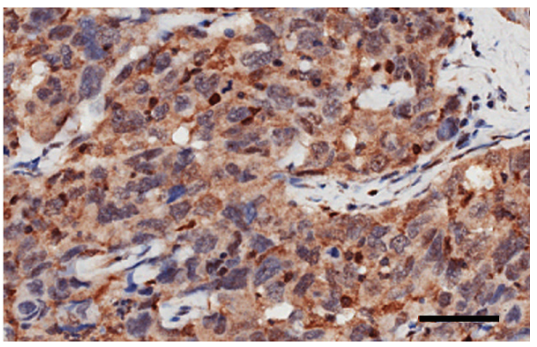

Adjacent nontumor
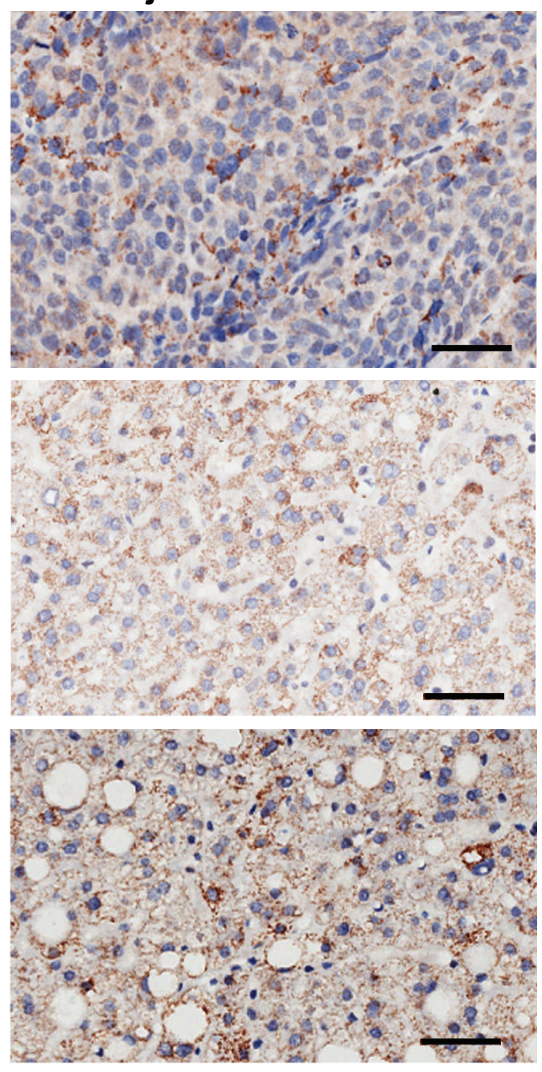
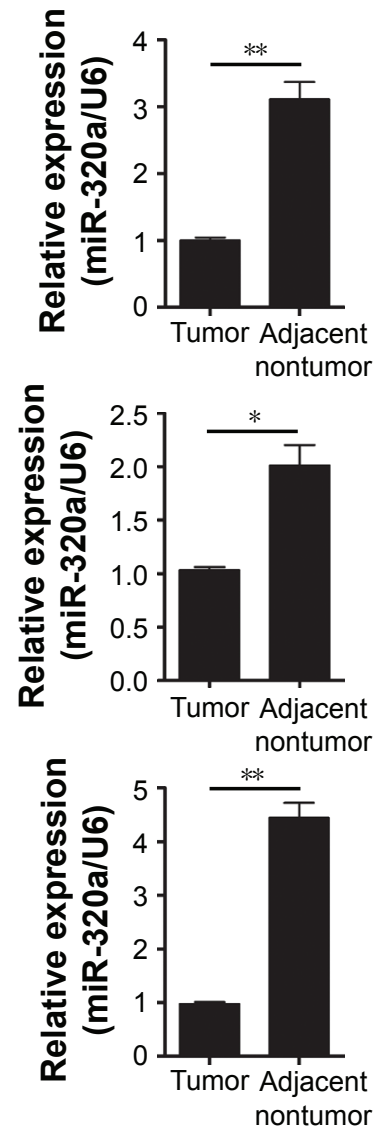

Figure 3 miR-320a downregulates endogenous c-Myc expression in HCC cells.

Notes: (A) The upregulation of miR-320a was verified by qRT-PCR after transfected with miR-320a mimics or NC in HepG2 and QGY-7703 cells. U6 was used as an internal control. (B) The protein levels of c-Myc expression were determined by Western blot after transfected with miR-320a mimics or NC in HepG2 and QGY-7703 cells. $\beta$-Actin was used as a control. (C) Analysis of c-Myc and miR-320a expression in the same HCC tissue by IHC and qRT-PCR. Brown signal in IHC was considered as positive staining for c-Myc. Scale bar $=50 \mu \mathrm{m}$. Data are presented as the mean \pm standard deviation $(n=3)$. Results were obtained in three replicates. $* P<0.05$, $* * P<0.0 \mathrm{I}$.

Abbreviations: HCC, hepatocellular carcinoma; IHC, immunohistochemistry; miR-320a, microRNA-320a; NC, negative control; qRT-PCR, quantitative reverse-transcription polymerase chain reaction.

protein kinase/extracellular signal-regulated kinase pathway. There are studies that proved that c-Myc is involved in cell growth, cell differentiation, apoptosis, invasion, and many other biological activities; ${ }^{32-34}$ thus, c-Myc is a very strong proto-oncogene. Pelengaris et al and Lin et al showed that the expression of C-Myc was found to be elevated in several types of cancers, such as HCC. ${ }^{35,36}$ Recent reports show that c-Myc targets miR-24, ${ }^{37}$ miR-145, ${ }^{38}$ let- $7 \mathrm{a},{ }^{39}$ and miR-185-3p. ${ }^{40}$ A possible regulation of c-Myc by miR-320a was previously shown by Lu et al. They showed that progesterone 
A

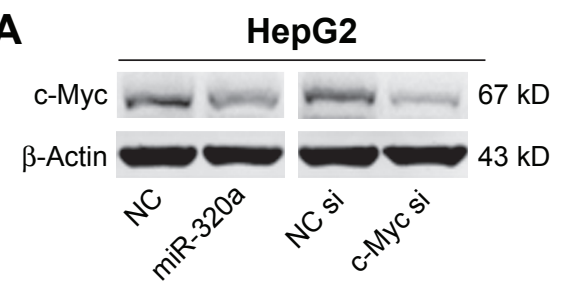

C

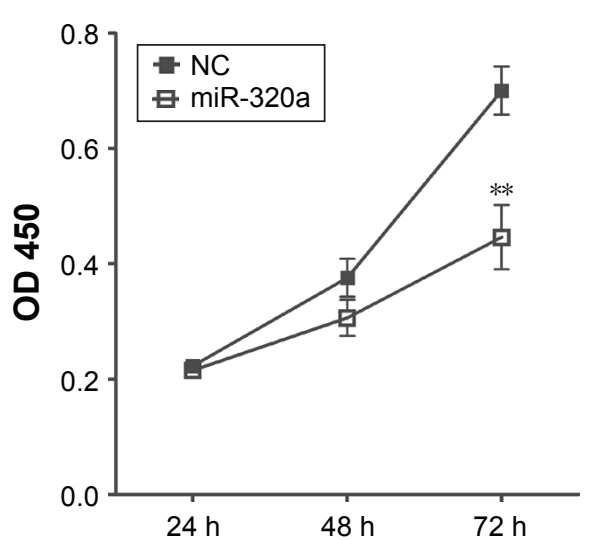

B QGY-7703

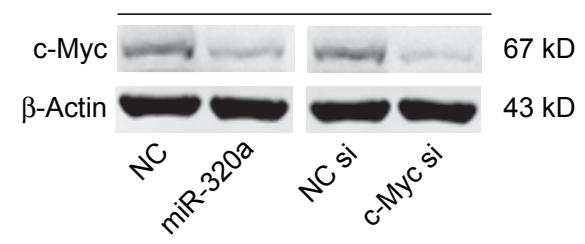

HepG2

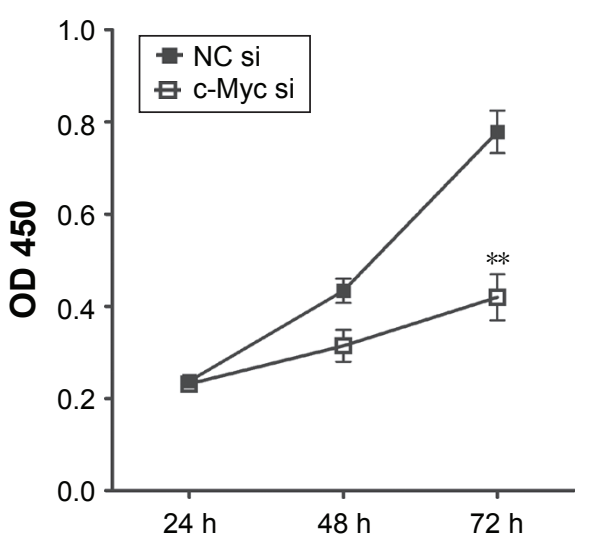

D

QGY-7703
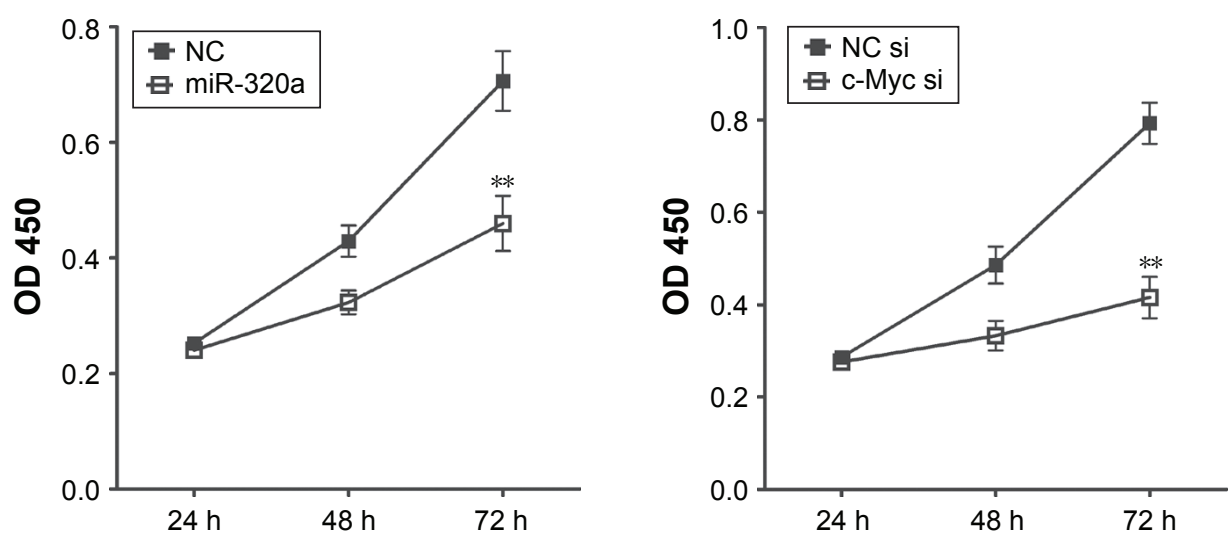

Figure 4 Upregulation of miR-320a inhibited c-Myc expression and hepatocellular carcinoma cells proliferation by targeting c-Myc in vitro.

Notes: After HepG2 and QGY-7703 cell lines treated with miR-320a mimics or c-Myc siRNA. Western blot assays of c-Myc protein were conducted in HepG2 (A) and QGY-7703 (B) cells and $\beta$-Actin was used as a control. 3-(4,5-Dimethylthiazol-2-yl)-2,5-diphenyl tetrazolium bromide assays were conducted in HepG2 cell line (C) and QGY-7703 cell line (D). Data are presented as the mean \pm standard deviation $(n=3)$. Results were obtained in three replicates. $* * P<0.01$.

Abbreviations: miR-320a, microRNA-320a; NC, negative control; OD, optical density; si or siRNA, small interfering RNA.

(P4) promoted the expression of miR-320a in MCF-7 cell line by repressing c-Myc expression, while estrogen (E2) exerted the opposite effect. ${ }^{23}$ However, correlation of c-Myc with miR-320a in HCC needs to be clarified. In this study, we found that c-Myc expression was upregulated by low expression of miR-320a in HCC tissues by IHC assay, and upregulation of miR-320a downregulated the c-Myc expression in HepG2 and QGY-7703 cell lines. Our results demonstrated that the c-Myc expression was inhibited by miR-320a in HCC tissues, HepG2, and QGY-7703 cell lines.

$\mathrm{Lu}$ et al found that the c-Myc/miR-320a axis provides a novel understanding of the mechanism of acquired tamoxifen resistance in BC. ${ }^{23}$ In this study, the biological underlying mechanisms of miR-320a correlated with c-Myc were investigated in HepG2 and QGY-7703 cells. We found that miR-320a mimics or c-Myc siRNA all lead to the similar decrease of c-Myc expression level, and the cell viability and proliferation were all decreased at the same time. We hypothesize that correlation of c-Myc with miR-320a may stimulate the HCC tumor development. Invasion is also a crucial feature in tumor development and tumorigenesis. Evidence showed that miR-320a may inhibit cell invasion in some ways. Zhang Y, et al, 2012 demonstrated that miR-320a may inhibit angiogenesis and cancer cell invasion 
A

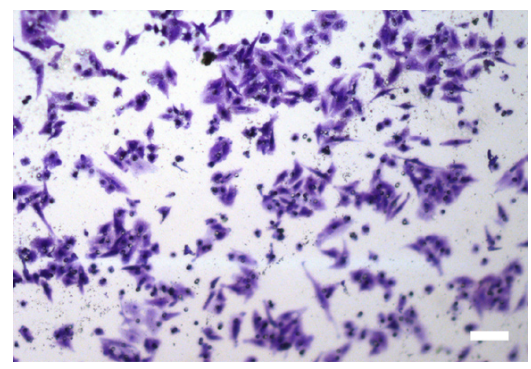

NC

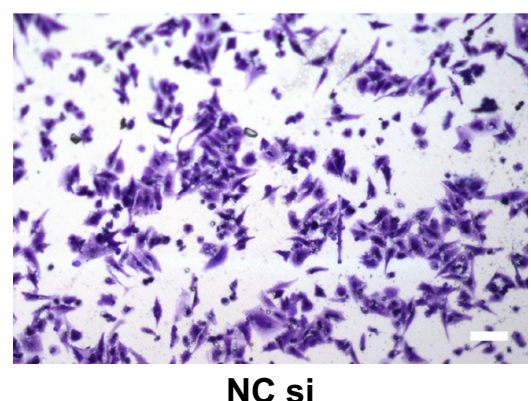

NC si

B
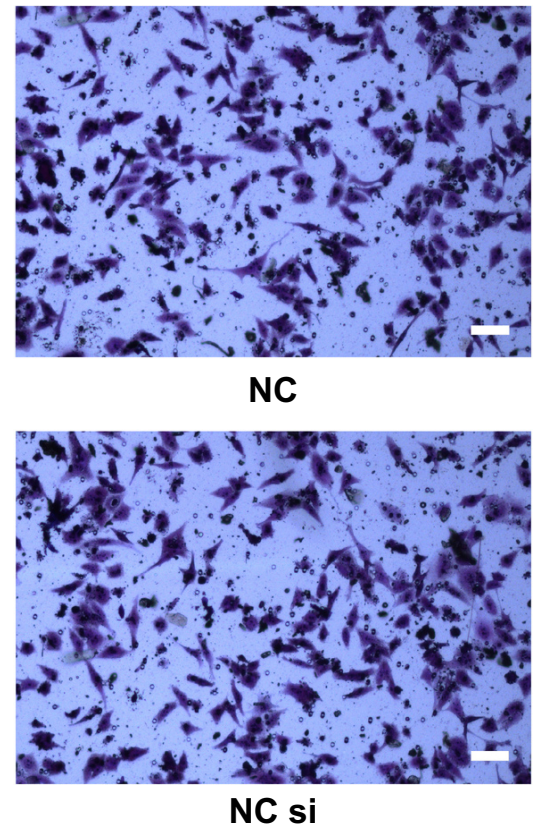

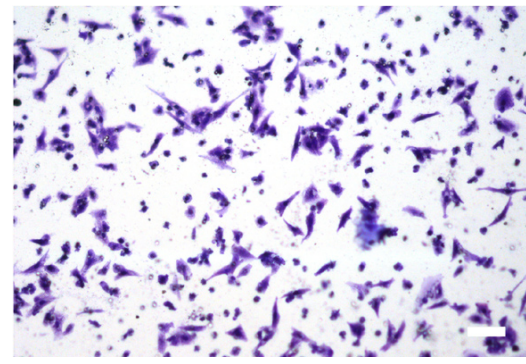

miR-320a
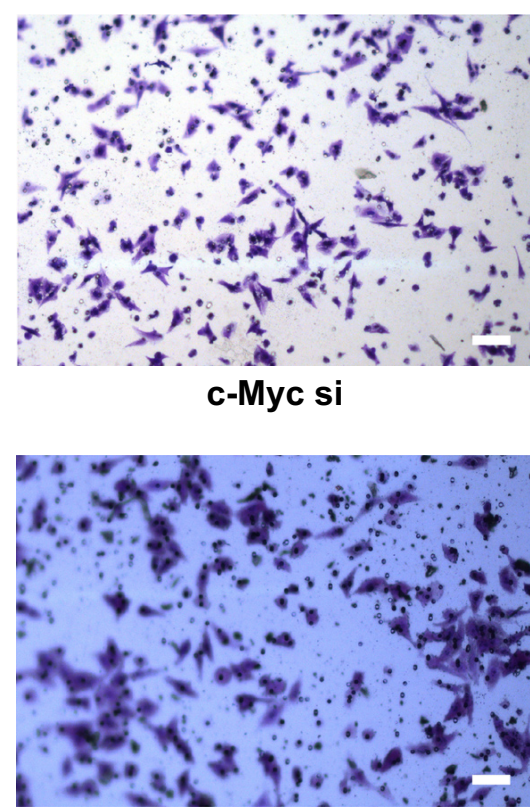

miR-320a

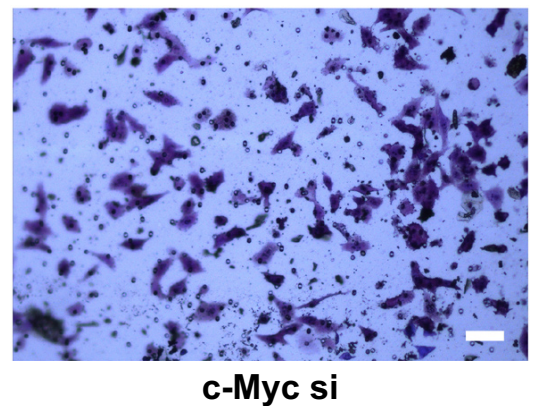

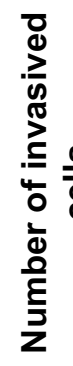
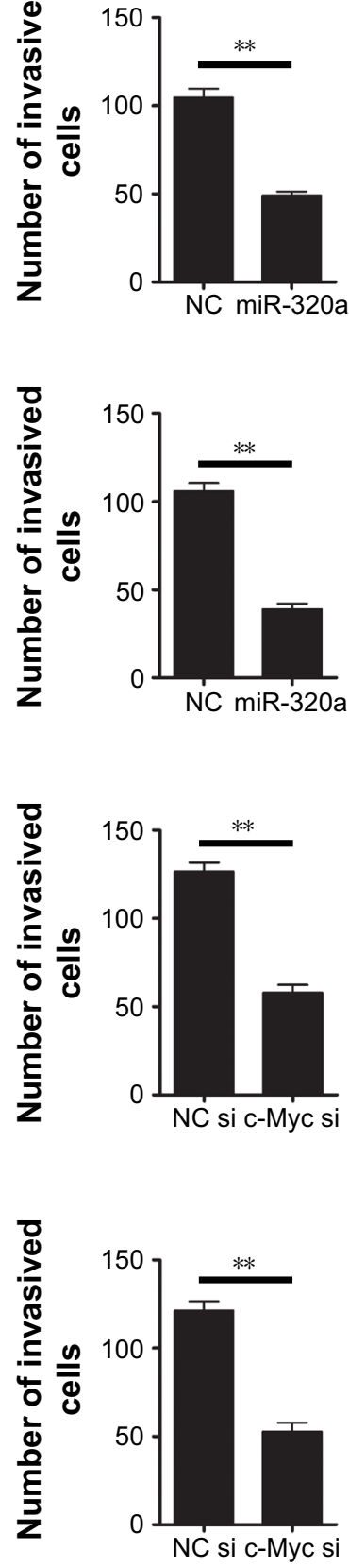

Figure 5 Upregulation of miR-320a inhibited hepatocellular carcinoma cells invasion by targeting c-Myc in vitro.

Notes: Transwell invasion assay was performed to detect the invasive properties in HepG2 (A) and QGY-7703 (B) cells after treated with miR-320a mimics or c-Myc siRNA; Scale bar $=50 \mu \mathrm{m}$. Data are presented as the mean \pm standard deviation $(n=3)$. Results were obtained in three replicates. $* * P<0.0 \mathrm{I}$.

Abbreviations: miR-320a, microRNA-320a; NC, negative control; si or siRNA, small interfering RNA.

by targeting NRP-1 in CRC. ${ }^{29}$ Nevertheless, the role of miR320 a by inhibiting c-Myc in HCC cells invasion remains unclear. Furthermore, our subsequent studies showed that the invasion capacity of HCC cells transfected with miR320a mimics or c-Myc siRNA was significantly lower than that of the HCC cells treated with respective controls. Taken together, our findings demonstrated that the proliferation and invasion effects of miR-320a were consistent with those of
c-Myc siRNA in HepG2 and QGY-7703 cell lines, implying that miR-320a represses the proliferation and invasion of HCC cell lines through targeting c-Myc.

\section{Conclusion}

In conclusion, miR-320a functions as a tumor suppressor through targeting c-Myc; our findings not only advance our understanding toward the molecular mechanism of 
carcinogenesis, but also provide evidence for further investigation that miR-320a acts as a novel biomarker or potential target for HCC treatment.

\section{Acknowledgments}

This work was financially supported by National Natural Science Foundation of China (No 81460435 and No 81372360), Key Project of Yunnan Applied Basic Research Plan (2014FA022), and Science Research Foundation of Education Department of Yunnan Province (2014Z004, 2014Y009).

\section{Disclosure}

The authors report no conflicts of interest in this work.

\section{References}

1. Hayes J, Peruzzi PP, Lawler S. MicroRNAs in cancer: biomarkers, functions and therapy. Trends Mol Med. 2014;20:460-469.

2. Marta GN, Garicochea B, Carvalho AL, Real JM, Kowalski LP. MicroRNAs, cancer and ionizing radiation: where are we? Revista da Associacao Medica Brasileira (1992). 2015;61(3):275-281. Portuguese.

3. Tutar L, Tutar E, Ozgur A, Tutar, Y. Therapeutic targeting of microRNAs in cancer: future perspectives. Drug Dev Res. 2015;76(7): 382-388.

4. Bao B, Wang Z, Ali S, et al. Metformin inhibits cell proliferation, migration and invasion by attenuating CSC function mediated by deregulating miRNAs in pancreatic cancer cells. Cancer Prev Res (Phila). 2012;5(3):355-364.

5. Chai J, Wang S, Han D, Dong W, Xie C, Guo H. MicroRNA-455 inhibits proliferation and invasion of colorectal cancer by targeting RAF proto-oncogene serine/threonine-protein kinase. Tumour Biol. 2015;36:1313-1321.

6. Chuang KH, Whitney-Miller CL, Chu CY, et al. MicroRNA-494 is a master epigenetic regulator of multiple invasion-suppressor microRNAs by targeting ten eleven translocation 1 in invasive human hepatocellular carcinoma tumors. Hepatology (Baltimore, Md.). 2015;62: 466-480.

7. Xie J, Tan ZH, Tang X, et al. MiR-374b-5p suppresses RECK expression and promotes gastric cancer cell invasion and metastasis. World $J$ Gastroenterol. 2014;20(46):17439-17447.

8. Jemal A, Bray F, Center MM, Ferlay J, Ward E, Forman D. Global cancer statistics. CA Cancer J Clin. 2011;61(2):69-90.

9. Tsoulfas G. Role of microRNA in the diagnosis and therapy of hepatocellular carcinoma: a new frontier. MicroRNA (Shariqah, United Arab Emirates). 2014;3(3):137-143.

10. Su H, Yang JR, Xu T, et al. MicroRNA-101, down-regulated in hepatocellular carcinoma, promotes apoptosis and suppresses tumorigenicity. Cancer Res. 2009;69(3):1135-1142.

11. Fornari F, Gramantieri L, Ferracin M, et al. MiR-221 controls CDKN1C/ p57 and CDKN1B/p27 expression in human hepatocellular carcinoma. Oncogene. 2008;27:5651-5661.

12. Wang Y, Lee ATC, Ma JZI, et al. Profiling microRNA expression in hepatocellular carcinoma reveals microRNA-224 up-regulation and apoptosis inhibitor-5 as a microRNA-224-specific target. J Biol Chem. 2008;283(19):13205-13215.

13. Xu T, Zhu Y, Xiong Y, Ge Y, Yun J, Zhuang S. MicroRNA-195 suppresses tumorigenicity and regulates G1/S transition of human hepatocellular carcinoma cells. Hepatology. 2009;50(1):113-121.

14. Gramantieri L, Ferracin M, Fornari F, et al. Cyclin G1 is a target of miR-122a, a microRNA frequently down-regulated in human hepatocellular carcinoma. Cancer Res. 2007;67(13):6092-6099.
15. Wong QW, Lung RW, Law PT, et al. MicroRNA-223 is commonly repressed in repatocellular carcinoma and potentiates expression of stathmin1. Gastroenterology. 2008;135(1):257-269.

16. Meng F, Henson R, Wehbe-Janek H, Ghoshal K, Jacob ST, Patel T. MicroRNA-21 regulates expression of the PTEN tumor suppressor gene in human hepatocellular cancer. Gastroenterology. 2007;133(2): 647-658.

17. Hou J, Lin L, Zhou W, et al. Identification of miRNomes in human liver and hepatocellular carcinoma reveals miR-199a/b-3p as therapeutic target for hepatocellular carcinoma. Cancer Cell. 2011;19(2):232-243.

18. Andersen HH, Duroux M, Gazerani P. MicroRNAs as modulators and biomarkers of inflammatory and neuropathic pain conditions. Neurobiol Dis. 2014;71:159-168.

19. Areeb Z, Stylli SS, Koldej R, et al. MicroRNA as potential biomarkers in Glioblastoma. J Neurooncol. 2015;125(2):237-248.

20. Krauskopf J, Verheijen M, Kleinjans JC, de Kok TM, Caiment F. Development and regulatory application of microRNA biomarkers. Biomark Med. 2015;9(11):1137-1151.

21. Dong Y, Zhao J, Wu CW, et al. Tumor suppressor functions of miR133a in colorectal cancer. Mol Cancer Res. 2013;11(9):1051-1060.

22. Vislovukh A, Kratassiouk G, Porto E, et al. Proto-oncogenic isoform A2 of eukaryotic translation elongation factor eEF1 is a target of miR-663 and miR-744. Br J Cancer. 2013;108(11):2304-2311.

23. Lu M, Ding K, Zhang G, et al. MicroRNA-320a sensitizes tamoxifenresistant breast cancer cells to tamoxifen by targeting ARPP-19 and ERRgamma. Sci Rep. 2015;5:8735.

24. Shang C, Zhang H, Guo Y, Hong Y, Liu Y, Xue Y. MiR-320a downregulation mediates bladder carcinoma invasion by targeting ITGB3. Mol Biol Rep. 2014;41(4):2521-2527.

25. Song My, Pan Kf, Su Hj, et al. Identification of serum microRNAs as novel non-invasive biomarkers for early detection of gastric cancer. PLoS One. 2012;7(3):e33608.

26. Wang H, Su X, Yang M, et al. Reciprocal control of miR-197 and IL-6/STAT3 pathway reveals miR-197 as potential therapeutic target for hepatocellular carcinoma. Oncoimmunology. 2015;4(10):e1031440.

27. Helwak A, Kudla G, Dudnakova T, Tollervey D. Mapping the human miRNA interactome by CLASH reveals frequent noncanonical binding. Cell. 2013;153(3):654-665.

28. Chen C, Wang Y, Yang S, et al. MiR-320a contributes to atherogenesis by augmenting multiple risk factors and down-regulating SRF. J Cell Mol Med. 2015;19(5):970-985.

29. Zhang Y, He X, Liu Y, et al. MicroRNA-320a inhibits tumor invasion by targeting neuropilin 1 and is associated with liver metastasis in colorectal cancer. Oncol Rep. 2012;27(3):685-694.

30. Yan LX, Huang XF, Shao Q, et al. MicroRNA miR-21 overexpression in human breast cancer is associated with advanced clinical stage, lymph node metastasis and patient poor prognosis. RNA (New York, N.Y.). 2008;14(11):2348-2360.

31. Schaar DG, Medina DJ, Moore DF, Strair RK, Ting Y. miR-320 targets transferrin receptor 1 (CD71) and inhibits cell proliferation. Exp Hematol. 2009;37(2):245-255.

32. Secombe J, Pierce SB, Eisenman RN. Myc: a weapon of mass destruction. Cell. 2004;117:153-156.

33. Adhikary S, Eilers M. Transcriptional regulation and transformation by Myc proteins. Nat Rev Mol Cell Biol. 2005;6(8):635-645.

34. Grandori C, Cowley SM, James LP, Eisenman RN. The Myc/Max/Mad network and the transcriptional control of cell behavior. Annu Rev Cell Dev Biol. 2000;16:653-699.

35. Pelengaris S, Khan M, Evan G. c-MYC: more than just a matter of life and death. Nat Rev Cancer. 2002;2(10):764-776.

36. Lin CP, Liu CR, Lee CN, Chan TS, Liu HE. Targeting c-Myc as a novel approach for hepatocellular carcinoma. World $J$ Hepatol. 2010;2(1):16.

37. Lal A, Navarro F, Maher CA, et al. MiR-24 inhibits cell proliferation by targeting E2F2, MYC, and other cell-cycle genes via binding to "seedless" 3'UTR microRNA recognition elements. Mol Cell. 2009; 35(5):610-625. 
38. Sachdeva M, Zhu S, Wu F, et al. p53 represses c-Myc through induction of the tumor suppressor miR-145. Proc Natl Acad Sci U S A. 2009; 106(9):3207-3212.

39. Sampson VB, Rong NH, Han J, et al. MicroRNA let-7a down-regulates MYC and reverts MYC-induced growth in Burkitt lymphoma cells. Cancer Res. 2007;67(20):9762-9770.
40. Liao JM, Lu H. Autoregulatory suppression of c-Myc by miR-185-3p. J Biol Chem. 2011;286(39):33901-33909.

\section{Publish your work in this journal}

OncoTargets and Therapy is an international, peer-reviewed, open access journal focusing on the pathological basis of all cancers, potential targets for therapy and treatment protocols employed to improve the management of cancer patients. The journal also focuses on the impact of management programs and new therapeutic agents and protocols on

\section{Dovepress}

patient perspectives such as quality of life, adherence and satisfaction. The manuscript management system is completely online and includes a very quick and fair peer-review system, which is all easy to use. Visit http://www.dovepress.com/testimonials.php to read real quotes from published authors.

\footnotetext{
Submit your manuscript here: http://www.dovepress.com/oncotargets-and-therapy-journal
} 